\title{
EFFECT OF CHEMOTHERAPY ON PHILADELPHIA CHROMOSOME IN CHRONIC MYELOID LEUKEMIA (CML) PATIENTS
}

\author{
Jha C B ${ }^{1}$, Kucheria $\mathrm{K}^{2}$, Chaudhary V $\mathrm{P}^{2}$
}

ABSTRACT

Chronic myeloid leukemia (CML) is a clonal myeloproliferative disorder as a result of neoplastic transformation of the primitive hemopoetic cells. It is well known that the Philadelphia chormosome (ph) is a specific abnormality found in $90 \%$ of CML patients. It has been reported that interferon has better effect on disease control and prognosis. Cytogenetic analysis of ph chromosome plays very important role in the prognosis and monitoring of therapy. In this present study 35 diagnosed patients of CML were considered, which included untreated patients of various age groups (262yrs). The cases were refered from haematology clinic of All India Institute of Medical Sciences (AIIMS). Out of 35 patients only 13 patients were available after six month of therapy for follow-up cytogenetic analysis. Out of 13 patient, 2 were ph negative, 8 were $100 \%$ ph positive and 3 were ph positive mosaic before therapy. Of the 3 mosaic patients, 2 remained unchanged after therapy and one patient became $100 \%$ ph negative. Though in general significant reduction in ph\% by interferon therapy was seen but minority patients showed complete cytogenetic remission.

Key Words: Chronic myeloid leukemia, chemotherapy, Philadelphia chromosome.

\section{INTRODUCTION}

Chronic myeloid leukemia is a clonal myeloproliferative disorder as a result of neoplastic transformation of the primitive haemopoietic stem cell. It accounts for about 7-15\% of all leukemias. ${ }^{1}$ Males are more commonly affected than female (14:1). ${ }^{2}$
The Philadelphia ( $\mathrm{ph}$ ) chromosome in the malignant cells is found in more than $90 \%$ of patients with CML. Nowell and Hungerford ${ }^{3}$ reported a very small chromosome that appeared to be a partially deleted copy of one of the two smallest autosome pairs. This small element became known as the Philadelphia chromosome. ${ }^{4}$

1. B. P. Koirala Institute of Health Sciences, Dharan, Nepal.

2. All India Institute of Medical Sciences.

Address for correspondence : Dr. Chandra Bhushan Jha

Assistant Professor

B.P.K.I.H.S., Dharan, Nepal.

Email: chandraj_2000@yahoo.com 
The genetics of CML plays very important role in the prognosis of patient's condition and for the monitoring of therapy, which are consistently associated with chromosome abnormality. Cytogenetic molecular analysis of the Philadelphia chromosome provide important information to the physician that are relevant to the leukemic burden of the patients.

It was planned to study chromosome in clinically diagnosed CML patients before and on chemotherapy.

\section{MATERIAL AND METHODS}

Thirty- five diagnosed cases of chronic myeloid leukemia were considered for this study, which included untreated patients of various age groups (2-62years). The diagnosis of CML was made on the basis of hematologic investigations, and cases were refered from hematology clinic of AIIMS.

\section{SAMPLE COLLECTION}

Bone marrow (BM) aspiration was done by the haematologist from the posterior superior iliac spine using heparinised syringe. $1 \mathrm{ml}$ of aspirate was collected in a sterile tube containing $5 \mathrm{ml}$ of RPMI 1640 (Gibco, BRL) culture media supplemented with 10-units/ $\mathrm{ml}$ of heparin. Samples were transported at room temperature.

\section{BONE MARROW CULTURE}

The bone marrow aspirate was centrifuged (1000 r.p.m; 10 minutes) and washed with RPMI 1640 media in the same tube in which it was transported. After discarding the supernatant, the pallet was supplemented in $5 \mathrm{ml}$ of RPMI 1640 reconstituted with $20 \%$ fetal bovine serum (Gibco, BRL). Two vials were set up for direct harvesting and for short-term culture for 24 hours in $\mathrm{Co}_{2}$ incubator at $37^{\circ}$ centigrade.

\section{CHROMOSOME PREPARATION}

Harvesting for bone marrow was done using colcemid (Gibco, BRL) treatment for 1 hour at a concentration of $0.2 / \mathrm{ml}$. The culture was centrifuged and the cell pallet was suspended in $10 \mathrm{ml}$ of hypotonic solution $(0.075 \mathrm{~m}$ pottassium chloride $(\mathrm{KCL})$ for 30 minutes at $37^{\circ} \mathrm{c}$. The suspension was again centrifuged and the cells were then fixed in methanol acetic acid fixative 3:1 for over night at $-20^{\circ} \mathrm{c}$. Precleaned slides were used for chromosome preparations. The cell suspension was dropped from an approximate distance of $40-50 \mathrm{~cm}$ on the slide placed at $30^{\circ}$. Conventional staining was done in 4\% Giemsa (Gibco, BRL) solution.

\section{GTG- BANDING}

On slide stored for 2-4 days at room temperature, GTG- banding was done using modified method of seabright5. Slide was then washed in normal saline and stained with $2 \%$ Giemsa (Gibco, BRL) stain.

\section{METAPHASE SCREENING}

The preparation was screened under X 10 objective using zeiss light microscope. Well spread metaphases were further analysed under x100 oil immersion objective. Minimum of 30 well spread metaphases were analysed. In samples showing absence or mosaicism for the Philadelphia chromosome 40-50 metaphases were screened. This was done to detect presence of few malignant cells and to ascertain the percentage of ph positive cells respectively. Two to five spread metaphases were photographed and karyotyped.

\section{MICROPHOTOGRAPHY AND KARYOTYPING}

Microphotography was done under x100 oil immersion using automatic exposure system of earl zeiss photo-micrographic equipment. The exposed 
film was developed with kodak's developer at $20^{\circ} \mathrm{c}$ using standard method. Prints were developed in

Fig. 1 standard print developer followed by fixation and washing. Karyotype analysis was done by cutting individual chromosomes from photographs of metaphase spreads (Plate land 2). The homologous pairs were arranged according to international system for cytogenetic nomenclature6on a predesigned format.

\section{RESULTS}

At the time of diagnosis, the cytogenetic analysis was done in all the cases and treatment stared with hydroxyurea(HU) or HU and interferon(IFN) combination chemotherapy (from 15 days- 2yrs duration). The following results were obtained.

\section{CYTOGENETIC ANALYSIS}

Fig.1. G - banded metaphase spread showing t $(9 ; 22)$ (q34, q11). Arrows indicate $9 q+$ and $22 q$.
All 35 patients were available for cytogenetic analysis at time of diagnosis, out of 35 patients, 17

Fig. 2 
were $100 \%$ ph positive $(49.9 \%), 10$ were $50-90 \%$ ph positive mosaicism $(28.5 \%)$ and 3 were $100 \%$ ph negative $(8.5 \%)$. In $14.25 \%$ of patients the cytogenetic analysis was failure at the time of diagnosis. $49.9 \%$ patients showed standard ph translocation9; $22\left(\mathrm{q}^{34} ; \mathrm{q}^{11}\right)$.

Out of 35 patients, only 13 were available for followup cytogenetic analysis after 6 months. At the time of diagnosis among these 13 patients, 8 were $100 \%$ ph positive, 3 were $50-90 \%$ ph positive mosaic and 2 were $100 \%$ ph negative. Two of the mosaic patients remained unchanged and one became $100 \%$ ph positive. Of the eight $100 \%$ ph positive patients, 5 became ph mosaic with ph positivity (50-70\%) and 2 patients remained unchanged, while one patients become $100 \%$ ph negative in combination chemotherapy (IFN and HU).

Cytogentic results showed better response with combination chemotherapy (IFN+HU). With cytogenetics results, total Leucocyte count (TLC) reduced and hemoglobin increased significantly after 3 months of therapy. There was significant changes at 3rd and 6th months in haematological parameters (Fig.3). The platelet count was not

Fig. 3 : Comparision of pre and On therapy analysis of peripheral blood samples

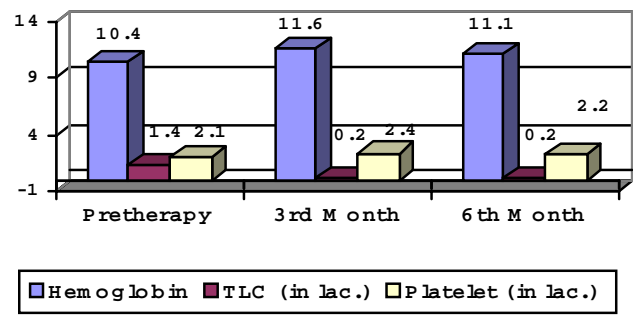

changed significantly after commencement of therapy, where as the liver and spleen were found reduced in size (Fig.4).

\section{Fig. 4 : Comparision of Liver and Spleen Size-pre and on Therapy}

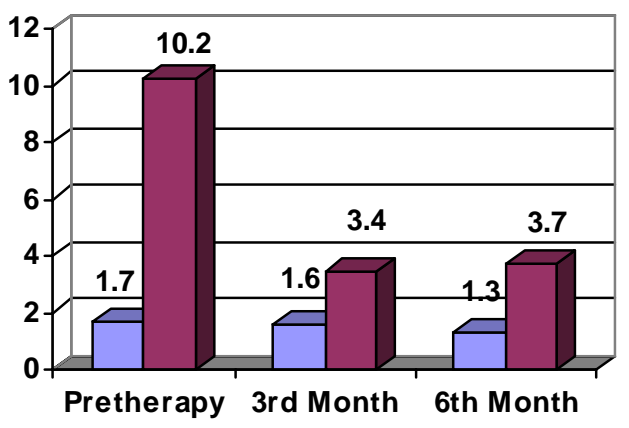

$\square$ Liver $\square$ Spleen

\section{DISCUSSION}

In the present study, out of 35 patients, only 13 patients were available for follow up cytogenetic analysis after 6 months. The cytogenetic hallmark of CML, the truncated chromosome No. 22 (ph chromosome) which is found due to translocation $t(9: 22)\left(q^{34} ; q^{11}\right)$ in $90-95 \%$ cases of CML. There have been various reports showing complete disappearance of ph chromosome following therapy with Busulfan or Hydroxyurea accompanied with prolonged haematologic remission. In this study, out of 13 patients 2 were ph negative, 8 were $100 \%$ ph positive and 3 were ph positive mosaic before therapy, of the 3 mosaic patients, 2 remained unchanged after therapy and one patients became $100 \%$ ph positive. Out of eight $100 \%$ ph positive patients, 5 became mosaic with ph positivity (50$70 \%$ ) and 2 patients remained unchanged, while one patients became $100 \%$ ph negative. Mosaicism in these patients may be transient and induced by treatment, m studying the ph status it has been demonstrated the uncomplicated ph in patients lymphnodes stimulated with epstein - Ban- virus. However, ph status was not studied in lymph nodes. Further, a ph reduction was considered good risk 
factor. $^{7}$ Thus, a study has shown a $30-50 \%$ ph reduction. A minority of patients with CML achieves a complete cytogenetic remission defined as disappearance of ph chromosome. ${ }^{8}$ In the present study one patients has shown complete disappearance of ph chromosome.

\section{REFERENCES}

1. Morrison VA. Chronic Leukemias. CA Cancer J din 1994; 44:353-377.

2. Hughes TP, Goldman JM. Chronic myeloid leukemia In : Hoffman R, Benz EJ , J r. Shattil SJ, et al ed. Hematology. Basic principles and practice. New York. Churchil livingstone; 1991: 854-869.

3. Nowell PC, Hungerford DA. A minute chromosome in human chronic granulocytic leukemia. Science 1960; 132:1497.
4. Baikie AG, Court Brown WM, Buckton K.E et al. A possible specific chromosome abnormality in human chronic myeloid leukemia. Nature 1960; 188:1165.

5. Seabright MA. A rapid banding technique for human chromosomes. Lancet 1971:2:971.

6. Hamden DG, Klinger HP In. ISCN. An international system for human cytogenetic nomenclature ed. New York: Karger, 1985.

7. Kloki 0 , Wandi U, Opalka B et al. A prospective randomized comparison of single agent interferon alpha with the combination of IFN-alpha and low dose $1 \mathrm{FN}$ - gamma in chronic myelogenous leukemia - EurJ Haematol 1992; 48:93-98.

8. Hochhaus A, Lin F, Reiter A et al. Variable numbers of BCR-ABL transcripts persist in CML patients who achieve complete cytogenetic remission with interferon alpha. Brit J Haem 1995; 91: 126.

\section{NMA wishes}

\section{to express sincere gratitude to}

\section{"Mathura Ratna Trust"}

(Founded by Late Dr. Raghubar Baidya and later augmented by

Late Dr. Bharat Raj Baidya in memory of their mother

Mathura Devi and father Dr. Ratna Das Baidya)

\section{for its contribution to publish this Journal}

